\title{
Her2-enriched breast cancer brain metastases exhibit resistance to Gamma Knife radiosurgery: findings from a single institutional series review
}

\author{
Jeffrey D. Radawski • Dongqing Zhang $\cdot$ Alex Nelson • \\ John C. Grecula • Nina A. Mayr • Nilendu Gupta • \\ John M. McGregor • Simon S. Lo • \\ Christopher E. Pelloski
}

Received: 24 January 2012 / Accepted: 20 April 2012 /Published online: 12 May 2012

(C) Springer-Verlag 2012

\begin{abstract}
Purpose/objective(s) This study aims to assess the outcomes and prognostic/predictive factors of overall survival and local control in patients with a diagnosis of brain metastasis from a breast cancer primary who underwent Gamma Knife radiosurgery (GKRS) as part of intracranial treatment.

Materials/methods A total of 69 patients were identified who were treated with GKRS for one to four brain metastases from breast cancer as a primary diagnosis. A total of 176 metastases were treated at diagnosis or as salvage treatment. Differences in overall survival time (OS) and local control time (LC) after GKRS between groups were assessed.

Results The median follow-up was 9.2 months (range 3-90.5 months). The median OS for this cohort was 13.4 months (range $0.5-90.5$ months). The median LC
\end{abstract}

J. D. Radawski · A. Nelson · J. C. Grecula • N. A. Mayr •

N. Gupta $\cdot$ C. E. Pelloski $(\triangle)$

Department of Radiation Oncology, Arthur G. James Cancer

Hospital, Ohio State University Medical Center,

300 W. 10th Ave., 094F James Cancer Center,

Columbus, OH 43210, USA

e-mail: christopher.pelloski@osumc.edu

D. Zhang

Department of Environmental and Radiological Health Sciences, Colorado State University,

Fort Collins, CO 80523, USA

J. M. McGregor

Department of Neurosurgery,

Ohio State University Medical Center,

Columbus, OH 43210, USA

S. S. Lo

Department of Radiation Oncology, University Hospitals Seidman Cancer Center, Case Western Reserve University,

Cleveland, $\mathrm{OH} 44106$, USA was 15.8 months. For a given metastasis, univariate analysis showed that LC was significantly correlated with the dose density (DD, ratio of GKRS dose to metastasis volume, [gray per cubic centimeters]). Additionally, human epidermal growth factor receptor 2 (HER2) enrichment predicted for worse local control on univariate analysis [HR 2.18; $95 \%$ CI 1.047-4.532] ( $p=0.037)$. With DD as a continuous variable, the only independent and significant predictive factors were the DD [HR: 0.99 ; 95\%CI: $0.984-0.998$ ] $(p=0.009)$ and HER2-enriched subtype $[\mathrm{HR}=2.25 ; 95 \%$ $\mathrm{CI}=1.074-4.728](p=0.032)$ after accounting for dose, metastasis volume, whole-brain radiotherapy (WBRT), and other subtypes. DD was then examined as a binary categorical variable whether or not a metastasis was treated to, at least, the median DD delivered across the cohort. With the cutoff set at this median, a DD value of $36 \mathrm{~Gy} / \mathrm{c}^{3}$, the independent and significant predictive factor again was the DD [HR: $3.49 ; 95 \% \mathrm{CI}: 1.45-8.40$ ] $(p=0.005)$ in favor of metastases receiving at least $36 \mathrm{~Gy} /$ $c^{3}$. Additionally, HER2 enrichment was significant when DD was examined as a binary variable, predicting for worse LC [HR 2.20; $95 \%$ CI: 1.048-4.636] $(p=0.037)$. The median LC for metastasis receiving $<36 \mathrm{~Gy} / \mathrm{c}^{3}$ is 18.7 months vs. median not reached in those receiving at least $36 \mathrm{~Gy} / \mathrm{c}^{3}(p<0.001)$. For HER2-enriched metastases, a DD $\geq 36 \mathrm{~Gy} / \mathrm{c}^{3}$ resulted in a $14 \%$ failure rate $(10$ month actuarial), compared to that of $0 \%$ observed in non-HER2-enriched subtypes.

Conclusion The ratio of dose delivered with GKRS to metastasis volume was significantly correlated with LC, with values $\geq 36 \mathrm{~Gy} / \mathrm{c}^{3}$ improving LC. This effect was significantly diminished in HER 2 subtypes, and a required higher DD for improved control, suggesting these metastases may be intrinsically resistant to radiosurgery. 
Keywords Brain metastasis · Breast cancer - Gamma knife · Radiosurgery

$\begin{array}{ll}\text { Abbreviations } \\ \text { DD } & \text { Dose density } \\ \text { ER } & \text { Estrogen receptor } \\ \text { GKRS } & \text { Gamma Knife radiosurgery } \\ \text { HER2 } & \text { Human epidermal growth factor receptor } 2 \\ \text { KPS } & \text { Karnofsky performance status } \\ \text { LC } & \text { Local control time } \\ \text { OS } & \text { Overall survival time } \\ \text { PR } & \text { Progesterone receptor } \\ \text { RPA } & \text { Recursive partitioning analysis } \\ \text { Rx } & \text { Prescription } \\ \text { WBRT } & \text { Whole-brain radiotherapy }\end{array}$

\section{Objectives}

The purpose of this study is to analyze the outcomes of patients with a diagnosis of brain metastasis from a breast cancer primary who underwent Gamma Knife radiosurgery (GKRS) as part of the treatment and to determine if there are any clinically available prognostic or predictive factors that should be considered when treating these patients.

\section{Background and rationale}

Breast cancer is the most common malignancy in females; approximately 182,000 new cases are estimated for 2008 [15]. Previous reports have estimated that $16 \%$ of breast cancer patients with metastatic disease develop clinically evident brain metastasis [11]. Systemic therapy options for patients with metastatic breast carcinoma have improved dramatically over the years [8]. However, the development of brain metastasis from breast cancer provides a significant challenge for these treatments. While there have been developments of chemotherapeutic agents better able to penetrate the blood-brain barrier, its role is investigational [22]. Local therapy, such as craniotomy with resection and/ or radiation therapy, remain as the primary modalities in the treatment of these patients.

Among the radiation therapy options, whole-brain radiotherapy (WBRT) remains an important modality of care [18], yet alternate options, such radiosurgery, provide effective control in a relatively noninvasive manner and are increasingly being utilized [27, 28]. Previous reports have identified dosing recommendations which minimize toxicity [23], and further reports have demonstrated benefits to improve local control in combining both WBRT and GKRS [2, 3]. Other investigations have seen improvements in breast cancer patients in terms of progression of brain metastasis and overall survival when examined retrospectively [4]. With the improved systemic options for breast cancer which have resulted in better control of extracranial disease, the survival of breast cancer patients is subsequently prolonged. The control of metastatic disease in the brain and neurotoxicity resulting from treatment of brain metastasis is important. It is beneficial to examine patient data and characteristics with regard to identifying potential prognostic factors for this subset of patients.

Other institutions have provided their results in such situations $[1,7,9,13,19,21]$. Within our institution, a respectable number of patients with brain metastasis from breast carcinoma have been treated with GKRS. We, therefore, have set out to formalize the Ohio State University experience in treating breast cancer patients with brain metastases utilizing GKRS.

\section{Materials and methods}

\section{Patient cohort}

Patients with a diagnosis of breast cancer and brain metastases treated at our institution with GKRS (Leksell Gamma Knife; Elekta Inc., Atlanta, GA) between March 1999 and January 2009 were identified. Exemption from the institutional review board at the Arthur G. James Cancer Hospital was obtained before the research was initiated. A total of 109 patients, all of whom were female, were identified. This spanned 128 treatment sessions. Thirty-eight patients with more than four brain metastases treated at the time of first radiosurgery were excluded for the purposes of this study, as were two additional patients who presented with metastases not in the brain parenchyma but within the skull or base of the skull. The remaining 69 patients presented initially with 129 brain metastases. However, these patients may have developed metastasis in a separate area salvaged with GKRS, and as such, these were analyzed as well. As such, a total of 176 metastases form the basis of this study; however, 41 metastases have been excluded from the analysis. Reasons for exclusion are that nine lesions received WBRT after failure for salvage and 32 did not have follow-up greater than 3 months.

Patients were classified into four major subtypes based on pathologic immunohistochemistry results of the primary pathology specimen's estrogen receptors (ER), progesterone receptors (PR), and human epidermal growth factor receptor 2 (HER2) status [24, 25]. Specifically, patients with ER positivity and/or PR positivity and HER 2 negativity were classified as luminal A subtype. Patients with ER positivity and/or PR positivity and HER2 positivity were classified as luminal B subtype. Patients with ER and PR negativity and 
HER2 positivity were classified as HER2-enriched subtype. Lastly, patients with ER, PR, and HER2 negativity were classified as basal subtype.

GKRS was performed in conjunction with a neurosurgeon within our department. Stereotactic headframe placement was performed by the neurosurgeon under local anesthesia on the morning of the procedure. A stereotactic MRI was subsequently performed with the headframe in-place and a thin-cut T1 axial MRI with double contrast or MultiHance was utilized to delineate metastasis volumes and perform planning. Volume delineation and planning was performed jointly between the radiation oncologist and neurosurgeon, and the number of lesions present was confirmed by a neuroradiologist. Volumes were delineated by outlining the contrast enhancement of the brain metastases, and total volume of a given metastasis was calculated with the Leksell treatment planning system. Planning involved conforming prescription dose to this volume as conformally as possible, typically maintaining $100 \%$ of the volume covered by the prescription dose. Characteristics were examined relating to treatment delivery, including minimum dose delivered to $100 \%$ of the metastatic volume, the volume of the metastasis, and the ratio of these two parameters which we will term the dose density (DD), with units of dose per cubic centimeter of volume [gray per cubic centimeter]. Specifically, this ratio is developed based on the radiobiologic principle that likelihood of complete metastasis eradication for a given dose is dependent on the number of cells (which correlates with metastasis volume) present at inception of treatment $[5,26]$. This metric is used to analyze the variety of radiation doses used clinically as well as the wide range of volumes in the treated metastasis.

Patients were followed every 3 months for the first 2 years after GKRS with the treating radiation oncologist. All these patients were also followed by their medical oncologists as part of the multidisciplinary care. These visits included an updated history and physical assessment of potential toxicities and a repeat MRI to evaluate treatment response. After 2 years, the follow-up intervals were typically lengthened to every 4-6 months if there was no evidence of intracranial progression.

\section{Statistical analysis}

Overall survival is determined from the date of GKRS to the date of death as obtained from either the medical record or social security database index or censored as of the date of last follow-up. Local recurrence is defined in accordance with criteria similar to RECIST 1.1 criteria [12]. Specifically, to score a local failure, there must be at least a $20 \%$ increase in the diameter of target lesions, taking as reference the smallest diameter found either at initial GKRS or on follow-up imaging. Additionally, an absolute increase of at least $0.5 \mathrm{~cm}$ is required in the diameter. Time to local recurrence is measured from the date of radiosurgery to the date of the above criteria or censored at last follow-up date.

Differences in overall survival time (OS) and local control time (LC) after GKRS between groups were assessed using a univariate logrank and Cox proportional hazard model [10]. Univariate survival curves were generated using the Kaplan-Meier method [16]. Independent factors were identified using multivariate Cox regression [10]. All analyses were performed using SPSS software (SPSS version 20, SPSS Incorporated, Chicago IL).

\section{Results}

Demographics/patient characteristics

Pertinent characteristics were recorded for this cohort of patients and displayed in Table 1 . The median age of patients treated was 50 (range $31-82$ ). The majority of patients were classified as recursive partitioning analysis (RPA) 2 (58 patients, $84 \%$ ). All patients had a KPS of at least 70 . The majority of patients presented with one metastasis (36 patients, $52 \%$ ). Patients presented with a variety of luminal subtypes, with the majority (24 patients, $35 \%$ ) being HER2-enriched. A total of nine patients were unable to have any of their receptors identified in the records and were classified as having an unknown subtype.

Clinical presentation of patients varied, and the most common symptoms prompting an MRI scan of the brain and diagnosis were headache (23 patients, $33 \%$ ) and nausea/vomiting (13 patients, $19 \%)$. Fourteen patients $(20 \%)$ were diagnosed without symptoms based on surveillance imaging.

\section{Treatment characteristics}

This cohort consists of patients with various treatment regimens prior to and after their initial GKRS. Table 2 contains information about the radiosurgery that patients underwent as well as additional and/or concurrent therapies that patients received with their radiosurgery. Patients were treated with varying radiosurgical doses. Specifically, 141 of the metastases were treated to at least an 18 Gy prescription dose, whereas the remaining 35 received less than this dose.

Patients underwent a variety of treatments prior, concurrent, and after initial radiosurgery. Surgical resection was typically employed for large and/or symptomatic metastases. WBRT was performed at some point in the majority of patients (48) with a median dose of 30 Gy in 15 fractions. The number of patients who were HER2-enriched or not and who had received WBRT or not was not statistically 
Table 1 Patient characteristics

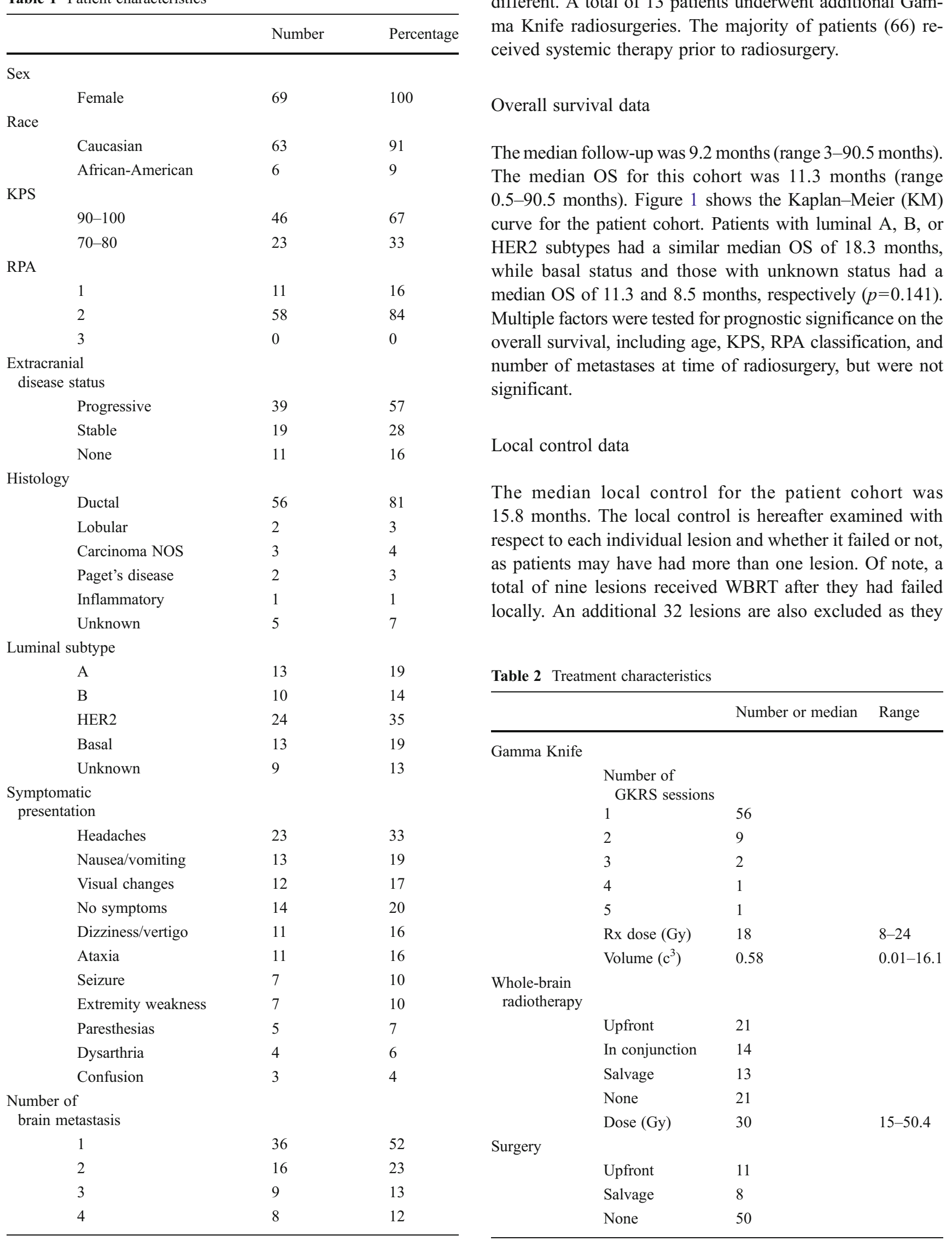

different. A total of 13 patients underwent additional Gamma Knife radiosurgeries. The majority of patients (66) re-

. 


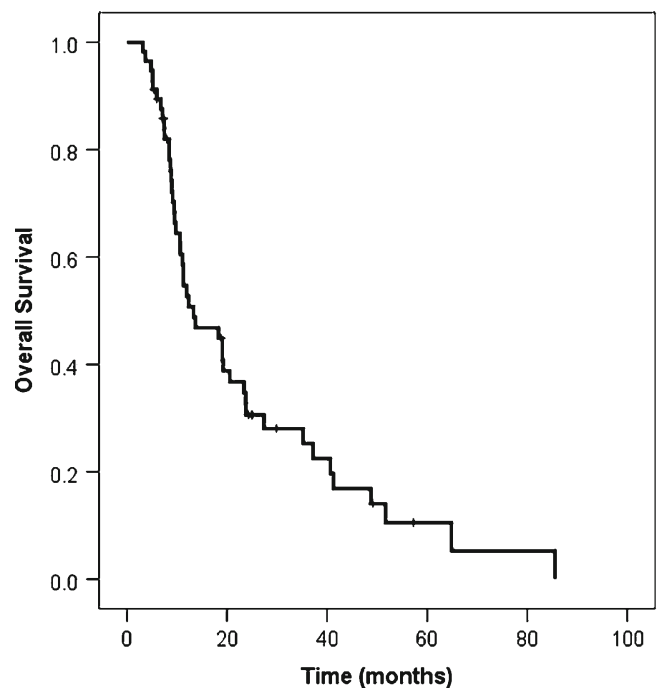

Fig. 1 Kaplan-Meier curves showing overall survival for the entire cohort

have less than 3 months of follow up time. As such, these lesions were excluded from the multivariate analyses, since in these cases, whole-brain treatment was administered in response to a measured endpoint or there was insufficient follow-up. A total of 135 of the initial 176 lesions (77\%) form the basis of the following results. On univariate analysis, the local control was significantly impacted by the DD [HR 0.99; 95\%CI 0.984-0.998] $(p=0.009)$ and was also significant on whether or not they were HER2-enriched [HR 2.18; $95 \%$ CI 1.047-4.532] ( $p=0.037)$. Figure 2 demonstrates the KM curve for local control for HER2-enriched metastases and all others. Median local control for the nonHER2-enriched patients was 48 months vs. 23.3 months for those that were HER2-enriched (logrank $p=0.032$ ). With DD as a continuous variable, the only independent and statistically significant predictive factors on multivariate analysis were the DD [HR: 0.99; 95\%CI: 0.986-0.999] $(p=0.016)$ and HER 2 enrichment $[\mathrm{HR}=2.25 ; 95 \%$ $\mathrm{CI}=1.074-4.728](p=0.032)$, after accounting for prescribed dose, volume of metastasis, and the delivery of WBRT.

To further illustrate the impact of DD, it is stratified into a categorical variable with values of either greater than or equal to $36 \mathrm{~Gy} / \mathrm{c}^{3}$ or less. This value was chosen as it represented the statistical median of all dose densities across the cohort. When DD is analyzed as a binary categorical variable, the independent and significant predictive factors, again, were the DD [HR: 3.49 ; 95\%CI: $1.45-8.40$ ] $(p=0.005)$ and HER enrichment [HR 2.20;95\% CI: $1.048-4.636]$ ( $p=0.037)$ after accounting for dose, volume, and whether or not WBRT was delivered. The KM curve for local control stratified by metastases receiving a DD of at least $36 \mathrm{~Gy} / \mathrm{c}^{3}$ or greater is presented in Fig. 3. The median local control for those metastases receiving at least $36 \mathrm{~Gy} / \mathrm{c}^{3}$ was not reached, and for those receiving less than $36 \mathrm{~Gy} / \mathrm{c}^{3}$

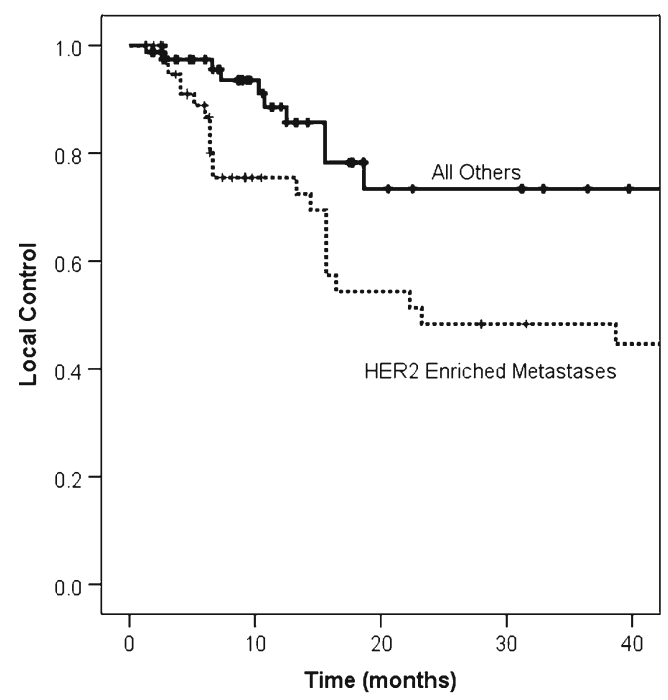

Fig. 2 Kaplan-Meier curve showing local control for metastases which were HER2-enriched compared to all others. Median local control for the non-HER2 enriched patients was 48 months vs. 23.2 months for those that were HER2-enriched (logrank $p=0.019$ )

was 18.7 months (logrank $p=0.001)$. To illustrate how these HER2 enrichment and DD impacted LC, comparisons were made between HER2-enriched and nonenriched groups after stratification by the median DD. For HER2-enriched metastases, a $\mathrm{DD} \geq 36 \mathrm{~Gy} / \mathrm{c}^{3}$ resulted in a $14 \%$ failure rate $(10$ month actuarial) compared to that of $0 \%$ observed in nonHER2 subtypes (logrank $p=0.005$ ). For HER2-enriched metastases, a DD $<36 \mathrm{~Gy} / \mathrm{c}^{3}$ resulted in a $33.5 \%$ failure rate (10-month actuarial) compared to that of $13.5 \%$ observed in the non-HER2 subtypes (logrank $p=0.245$ ).

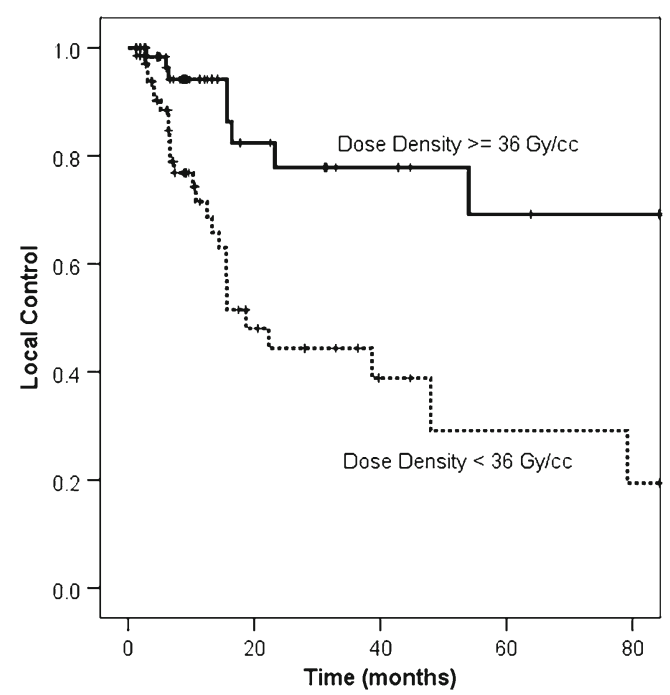

Fig. 3 Kaplan-Meier curve showing local control for each treated brain metastasis stratified by whether the dose density received was greater than or equal to $36 \mathrm{~Gy} / \mathrm{c}^{3}$ or not. Median local control for those metastases receiving at least $36 \mathrm{~Gy} / \mathrm{c}^{3}$ was not reached, and for those receiving less than $36 \mathrm{~Gy} / \mathrm{c}^{3}$ was 18.7 months $(\operatorname{logrank} p=0.001)$ 
Fig. 4 Kaplan-Meier curves stratified by metastases receiving a at least $36 \mathrm{~Gy} / \mathrm{c}^{3}$ or b less than $36 \mathrm{~Gy} / \mathrm{c}^{3}$. Each stratification is further separated by whether or not metastases were HER2 enriched (dotted) or not (solid). The 10-month actuarial failure rates in a were $14 \%$ in HER2-enriched patients and $0 \%$ in non-HER2enriched patients (logrank $p=$ 0.005). The 10-month actuarial failure rates in b were $33.5 \%$ in the HER2-enriched patients and $13.5 \%$ in non-HER2-enriched patients $(\operatorname{logrank} p=0.242)$

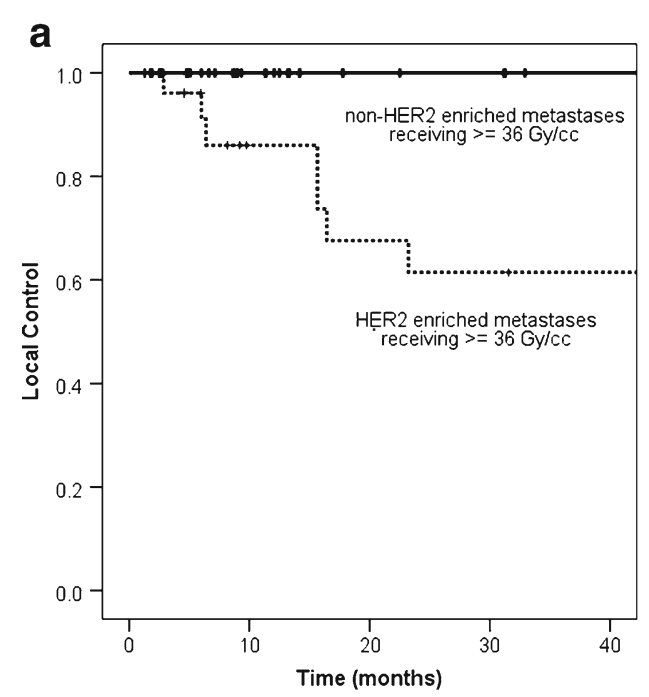

The corresponding LC curves demonstrating this are shown in Figs. $4 a$ and $b$.

\section{Toxicity}

Patients, overall, tolerated radiosurgery relatively well. Peak RTOG toxicities for patients were graded as level I or II for 55 of the 69 patients. A total of ten patients developed RTOG grade III toxicities as their peak toxicity. One patient developed level IV toxicity. Follow-up was not sufficiently long for three patients to be able to comment on their toxicity.

For the ten patients who developed peak grade III toxicities, the median time to toxicity was 7 months (standard deviation 9 months, range $0.8-28$ months). For eight of these ten patients, toxicities were judged to be, in the most part, related to metastasis progression, whereas for two of these patients, toxicities were judged to be related to radiosurgery. Six of the patients developed ataxia, two developed seizures, one developed focal weakness, and one developed decreased vision. Most of these patients responded favorably to steroid therapy with dexamethasone.

The patient who developed level IV toxicity was found with confusion, mental status changes, and concern for radiographic progression in the larger metastasis on MRI scan. Her initial CNS metastasis presentation consisted of a solitary metastasis and was treated with surgical resection followed by WBRT to a dose of 30 Gy in 12 fractions. Her first radiosurgery occurred 1 year after WBRT was completed and included two treated and neighboring metastases. The larger volume treated was $9.6 \mathrm{c}^{3}$ to a total of 18 Gy to the $50 \% \mathrm{IDL}$, which encompassed the metastasis. The smaller volume measured $0.29 \mathrm{c}^{3}$ and was treated to the same parameters. Her level IV toxicity rose 22.5 months after initial GKRS. She underwent surgical resection of the treated lesion where pathologic examination of the resected enlarging mass revealed necrosis and fibrosis without evidence of metastasis. Postoperatively, she returned to a relatively asymptomatic state and was followed for 41.7 months after initial radiosurgery.

\section{Discussion}

Review of our institutional experience of GKRS in the treatment of breast cancer brain metastases has given some insights into potentially important factors predicting for local control, namely, DD and the HER2-enriched subtype. We have demonstrated from our cohort that the DD is a predictive factor when analyzed on a continuous scale. For every additional gray per cubic centimeter of metastasis able to be delivered, the hazard ratio for local control improves approximately $1 \%$. The local control was vastly improved for metastases over the median DD of $36 \mathrm{~Gy} / \mathrm{c}^{3}$, with median control not reached in those above this DD compared to 18.7 months in those metastases not able to achieve this DD. The KM curves dramatically demonstrate this difference in local control well in Fig. 3.

Additionally, HER2-enriched metastases predicted for worse local control when compared to all other subtypes. These HER2-enriched metastases had significantly worse 10-month actuarial local control when compared to their non-HER 2 counterparts at $86 \%$ control compared to $100 \%$ control, even when stratified relative to DD more than $36 \mathrm{~Gy} / \mathrm{c}^{3}$. Additionally, the presence of HER2-enriched metastases predicted for inferior local control on a univariate analysis with a hazard ratio of 2.18 and to a similar statistically significant degree on multivariate analysis. The KM curve demonstrates this difference in local control well in Fig. 2.

This data seems to add support to data that suggests the HER-2 subtype may be inherently radioresistant. There has been considerable debate regarding the radioresistance of 
HER2-enriched tumors. The Danish group recently published their data examining the impact of receptor status on local regional control for patients who were enrolled on the $82 \mathrm{~b}$ and $82 \mathrm{c}$ studies [17]. Interestingly, for patients who were HER2-enriched, the risk of LRR was not diminished by radiotherapy ( $21 \%$ vs. $33 \%, p=0.200)$. Additionally, similar support is found within patients undergoing breast conservation treatment at Harvard University (HattangadiGluth et al.; [14]). These studies have been performed either post hoc or retrospectively though, and the level of evidence must be accordingly placed given this fact. Additionally, these studies are somewhat more difficult to interpret given the effect of targeted systemic therapy.

Contrary to the above reports, there are also other retrospective reports which do not support the rationale that HER2 tumors may confer radioresistance. The MD Anderson experience seemed to suggest no excess of failures from their retrospective analysis [6]. Additionally, further retrospective evidence was recently reported by Cleveland Clinic which seems to lend support that OS may be better in HER2-positive patients despite the potential radioresistance [29]. Interestingly, our OS data would possibly support this, although due to the more limited patient numbers, there was only enough power to examine luminal $\mathrm{A}$ and $\mathrm{B}$, and HER2 patients compared to those with basal or unknown subtypes and is of limited interpretability.

Clearly additional prospective data is needed to help answer the question of the impact of receptor status and its relation to prognosis and response to therapy. Yet in the mean time, our institution's findings seem to support the idea of HER2-enriched tumors as radioresistant. An inherent weakness of our study is its retrospective nature, with intrinsic difficulties therein. Additionally, it does have relatively small numbers of patients. Yet, it is intriguing to note though that in this series, where tumors are given ablative doses of radiotherapy in an anatomic location in which systemic therapy has relatively little impact, HER2 enrichment confers some degree of increased local failure relative to its non-HER2 enriched counterparts. Lastly, it is important for clinicians to note the distinction between the HER2enriched patients and those of luminal B status in whom there may be HER2 expression. Explicitly, the HER2enriched patients described above are ER- and PR-negative.

In conclusion, we feel that our data does suggest a dosevolume-response relationship in breast cancer metastatic to the brain treated with GKRS. The optimal dose-volume relationship is difficult to define given our patient cohort limits statistical power. However, for every additional $1 \mathrm{~Gy} / \mathrm{c}^{3}$ of tumor, an additional $1 \%$ increase in local control is achieved. Further, doses delivered of, at least, the median DD achieved in our series of $36 \mathrm{~Gy} / \mathrm{c}^{3}$ of tumor suggest significantly improved local control than their counterparts that are unable to achieve this dose level. Additionally, we have demonstrated that in our cohort, those with HER2-enriched tumors had relative resistance to GKRS even at dose densities exceeding $36 \mathrm{~Gy} / \mathrm{c}^{3}$. Further attention and study are needed to more clearly define the dose-volume relationship and the role of hormonal receptor status as it relates to prognosis and selection of therapy.

Conflicts of interest The authors do not have any conflicts of interest to disclose.

\section{References}

1. Amendola Be, Wolf Al Coy Sr, Amendola M, Bloch L (2000) Gamma knife radiosurgery in the treatment of patients with single and multiple brain metastases from carcinoma of the breast. Cancer J 6(2):88-92

2. Andrews Dw, Scott Cb, Sperduto Pw, Flanders Ae, Gaspar Le, Schell Mc, Werner-Wasik M, Demas W, Ryu J, Bahary Jp, Souhami L, Rotman M, Mehta Mp, Wj Curran Jr (2004) Whole brain radiation therapy with or without stereotactic radiosurgery boost for patients with one to three brain metastases: phase III results of the RTOG 9508 randomised trial. Lancet 363(9422):1665-1672

3. Aoyama H, Shirato H, Tago M, Nakagawa K, Toyoda T, Hatano K, Kenjyo M, Oya N, Hirota S, Shioura H, Kunieda E, Inomata T, Hayakawa K, Katoh N, Kobashi G (2006) Stereotactic radiosurgery plus whole-brain radiation therapy vs. stereotactic radiosurgery alone for treatment of brain metastases: a randomized controlled trial. Jama 295(21):2483-2491

4. Bartsch R, Fromm S, Rudas M, Wenzel C, Harbauer S, Roessler K, Kitz K, Steger Gg, Weitmann Hd, Poetter R, Zielinski Cc, Dieckmann K (2006) Intensified local treatment and systemic therapy significantly increase survival in patients with brain metastases from advanced breast cancer-a retrospective analysis. Radiother Oncol 80(3):313-317

5. Brenner $\mathrm{Dj}$ (1993) Dose, volume, and tumor-control predictions in radiotherapy. Int J Radiat Oncol Biol Phys 26(1):171-179

6. Buchholz Ta, Huang Eh, Berry D, Pusztai L, Strom Ea, Mcneese Md, Perkins Gh, Schechter Nr, Kuerer Hm, Buzdar Au, Valero V, Hunt Kk, Hortobagyi Gn, Sahin Aa (2004) Her2/neu-positive disease does not increase risk of locoregional recurrence for patients treated with neoadjuvant doxorubicin-based chemotherapy, mastectomy, and radiotherapy. Int J Radiat Oncol Biol Phys 59 (5):1337-1342

7. Chang Sd, Lee E, Sakamoto Gt, Brown Np Adler Jr (2000) Jr. Stereotactic radiosurgery in patients with multiple brain metastases. Neurosurg Focus 9(2):e3

8. Chia Sk, Speers Ch, D'yachkova Y, Kang A, Malfair-Taylor S, Barnett J, Coldman A, Gelmon Ka, O'reilly SE, Olivotto Ia (2007) The impact of new chemotherapeutic and hormone agents on survival in a population-based cohort of women with metastatic breast cancer. Cancer 110(5):973-979

9. Combs Se, Schulz-Ertner D, Thilmann C, Edler L, Debus J (2004) Treatment of cerebral metastases from breast cancer with stereotactic radiosurgery. Strahlenther Onkol 180(9):590-596

10. Cox Dr, Oakes D (1984) Analysis of survival data. Chapman and Hall, New York

11. Distefano A, Yong Yap Y, Hortobagyi Gn, Blumenschein Gr (1979) The natural history of breast cancer patients with brain metastases. Cancer 44(5):1913-1918

12. Eisenhauer Ea, Therasse P, Bogaerts J, Schwartz Lh, Sargent D, Ford R, Dancey J, Arbuck S, Gwyther S, Mooney M, Rubinstein L, Shankar L, Dodd L, Kaplan R, Lacombe D, Verweij J (2009) 
New response evaluation criteria in solid tumours: revised RECIST guideline (version 1.1). Eur J Cancer 45(2):228-247

13. Firlik Ks, Kondziolka D, Flickinger Jc, Lunsford Ld (2000) Stereotactic radiosurgery for brain metastases from breast cancer. Ann Surg Oncol 7(5):333-338

14. Hattangadi-Gluth Ja, Wo Jy, Nguyen Pl, Abi Raad Rf, Sreedhara M, Niemierko A, Freer Pe, Georgian-Smith D, Bellon Jr, Wong Js, Smith $\mathrm{Bl}$, Harris Jr, Taghian Ag. Basal subtype of invasive breast cancer is associated with a higher risk of true recurrence after conventional breast-conserving therapy. Int J Radiat Oncol Biol Phys.

15. Jemal A, Siegel R, Ward E, Hao Y, Xu J, Murray T, Thun Mj (2008) CA Cancer J Clin 58(2):71-96

16. Kaplan El, Meier P. Nonparametric estimation from incomplete observations. J Am Stat Assoc (53):457-481, 1958.

17. Kyndi M, Sorensen Fb, Knudsen H, Overgaard M, Nielsen Hm, Overgaard J (2008) Estrogen receptor, progesterone receptor, HER-2, and response to postmastectomy radiotherapy in highrisk breast cancer: the Danish Breast Cancer Cooperative Group. J Clin Oncol 26(9):1419-1426

18. Lo Ss, Chang El, Suh Jh (2005) Stereotactic radiosurgery with and without whole-brain radiotherapy for newly diagnosed brain metastases. Expert Rev Neurother 5(4):487-495

19. Muacevic A, Kreth Fw, Tonn Jc, Wowra B (2004) Stereotactic radiosurgery for multiple brain metastases from breast carcinoma. Cancer 100(8):1705-1711

20. Nguyen Pl, Taghian Ag, Katz Ms, Niemierko A, Abi Raad Rf, Boon Wl Bellon Jr, Wong Js, Smith Bl Harris Jr (2008) Breast cancer subtype approximated by estrogen receptor, progesterone receptor, and HER-2 is associated with local and distant recurrence after breast-conserving therapy. J Clin Oncol 26(14):2373-2378

21. Petrovich Z, Yu C, Giannotta S1, O'day S, Apuzzo Ml (2002) Survival and pattern of failure in brain metastasis treated with stereotactic gamma knife radiosurgery. J Neurosurg 97(5 Suppl):499-506
22. Sharma M, Abraham J (2007) CNS metastasis in primary breast cancer. Expert Rev Anticancer Ther 7(11):1561-1566

23. Shaw E, Scott C, Souhami L, Dinapoli R, Kline R, Loeffler J, Farnan N (2000) Single dose radiosurgical treatment of recurrent previously irradiated primary brain tumors and brain metastases: final report of RTOG protocol 90-05. Int J Radiat Oncol Biol Phys 47(2):291-298

24. Sorlie T, Perou Cm, Tibshirani R, Aas T, Geisler S, Johnsen H, Hastie T, Eisen Mb, Van De Rijn M, Jeffrey Ss, Thorsen T, Quist H, Matese Jc, Brown Po, Botstein D, Eystein Lonning P, BorresenDale Al (2001) Gene expression patterns of breast carcinomas distinguish tumor subclasses with clinical implications. Proc Natl Acad Sci USA 98(19):10869-10874

25. Sorlie T, Tibshirani R, Parker J, Hastie T, Marron Js, Nobel A, Deng S, Johnsen H, Pesich R, Geisler S, Demeter J, Perou Cm, Lonning Pe, Brown Po, Borresen-Dale Al, Botstein D (2003) Repeated observation of breast tumor subtypes in independent gene expression data sets. Proc Natl Acad Sci USA 100 (14):8418-8423

26. Thames Hd, Suit Hd (1986) Tumor radioresponsiveness versus fractionation sensitivity. Int J Radiat Oncol Biol Phys 12(4):687691

27. Tsao M, Xu W, Sahgal A. A meta-analysis evaluating stereotactic radiosurgery, whole-brain radiotherapy, or both for patients presenting with a limited number of brain metastases. Cancer, 2011.

28. Tsao Mn, Rades D, Wirth A, Lo Ss, Danielson Bl, Gaspar Le, Sperduto Pw, Vogelbaum Ma, Radawski Jd, Wang Jz, Gillin Mt, Mohideen N, Hahn Ca, Chang El. Radiotherapeutic and surgical management for newly diagnosed brain metastasis(es): an American Society for Radiation Oncology evidence-based guideline. Practical Radiation Oncology, 2012.

29. Xu Z, Marko Nf, Chao St, Angelov L, Vogelbaum Ma, Suh Jh, Barnett Gh, Weil Rj. Relationship between HER2 status and prognosis in women with brain metastases from breast cancer. Int $\mathrm{J}$ Radiat Oncol Biol Phys, 2011. 